\title{
Fiber optic interferometric hydrophone using fiber Bragg grating with time division multiplexing
}

\author{
Chiaki Okawara* and Kenji Saijyou \\ Technical Research and Development Institute, Japan Defense Agency \\ 3-13-1, Nagase, Yokosuka, 239-0826 Japan
}

(Received 3 April 2006, Accepted for publication 8 July 2006)

Keywords: Fiber optic sensor, Hydrophone, Fiber Bragg Grating, Time division multiplexing PACS number: 43.38.Zp, 43.30.Xm [doi:10.1250/ast.28.39]

\section{Introduction}

Fiber optic hydrophones have advantages over conventional electroacoustic hydrophones (piezoceramic); that is, they are light, highly reliable, electrically passive (system requires no electrical components in the wet-end) and immune to electromagnetic interference [1-4]. In 1990, a Fabry-Perot interferometer using pairs of fiber Bragg gratings (FBGs) as reflectors was proposed [5]. The use of FBGs as reflectors in interferometric hydrophones greatly simplifies the construction of the distributed chain of the hydrophones by eliminating the required large number of couplers, joints, and other interconnects in other types of hydrophones.

We have demonstrated the usefulness of this FBG-type interferometric hydrophone [6]. One particularly interesting application of this hydrophone is an underwater acoustic sonar system (less than several $\mathrm{kHz}$ ). This application requires acoustic arrays comprising several hundred hydrophones. When designing a multiplexing scheme, the optical power distribution to hydrophones and maintaining the performance of a multiplexed hydrophone comparable to that of a single hydrophone must be considered [7]. To maximize the number of multiplexed hydrophones, the reflectance distribution must be designed to reflect identical optical power pulses from the hydrophones.

Therefore, we propose a method of designing the reflectance distribution of FBGs with a time division multiplexing (TDM) array configuration and demonstrate a TDM array with two hydrophones, which is the basis for multiplexing.

\section{Principle of operation}

The measurement setup used in this study is shown in Fig. 1. Repetitive pulses are generated using a laser. A delay coil is incorporated between hydrophones to ensure that individual pulses returning from each hydrophone do not overlap at the output. The fiber length between FBGs in hydrophones is set to be equal to the difference in fiber length between the arms of the compensator. Therefore, the return signal of the first pulse from $\mathrm{FBG}_{\mathrm{X} 1}\left(\mathrm{FBG}_{\mathrm{Y} 1}\right)$ and that of the second pulse from $\mathrm{FBG}_{\mathrm{X} 2}\left(\mathrm{FBG}_{\mathrm{Y} 2}\right)$ are coupled via a compensating interferometer that imposes a sinusoidal modulation by a piezostretcher. At the optical-electrical $(\mathrm{O} / \mathrm{E})$ converter, the intensity of the homodyne pulse, which is

\footnotetext{
*e-mail: okawara@jda-trdi.go.jp
}

recombined and modulated, is given by

$$
J=I_{0}\left(1+V \cos \left(C \cos \omega_{0} t+\phi(t)\right)\right),
$$

where $I_{0}$ is the total mean intensity of the homodyne pulse and $V$ is the fringe visibility term. $\omega_{0}$ and $C$ are the frequency and amplitude of sinusoidal modulation imposed on the compensating interferometer by the piezostretcher. $\phi(t)$ is the acoustic signal obtained by homodyne demodulation [8].

The fringe visibility term, which depends on the state of polarization of the pulses from each FBG, varies unpredictably and independently. In the worst case, where the fringe visibility term is zero, the acoustic signal is extinguished [9]. This is called polarization-induced signal fading (PSF). However, we do not consider this phenomenon in this study.

\section{Reflectance distribution design method for FBGs}

Here, we consider the reflectance distribution of FBGs to maximize the number of hydrophones in an array, which is indispensable for designing a multiplexing scheme. A schematic configuration of a typical multiplexed hydrophone arrangement is shown in Fig. 2(a). The array comprises a number of hydrophones, labeled one to $n$, spliced in series. For simplicity, the fiber length between FBGs is maintained equal: therefore, the $2 n$ FBGs regularly distributed in space are in series on a single fiber. The optical crosstalk due to the multiple reflections naturally occurring in the system is negligibly small. If the power launched into the $(n-1)$ th FBG $\left(\mathrm{FBG}_{n-1}\right)$ is unity, the returning powers from $\mathrm{FBG}_{n-1}$ and $\mathrm{FBG}_{n}$ to the laser side of $\mathrm{FBG}_{n-1}$ are expressed as $r_{n-1}$ and $r_{n}\left(1-r_{n-1}\right)^{2} \alpha^{2} \beta^{2}\left(r_{n}=10^{R_{n} / 10}, \alpha=10^{A / 10}, \beta=10^{B / 10}\right)$, respectively, where $R_{n}$ is the reflectance of $\mathrm{FBG}_{n}, A$ is the transmission loss between the FBGs and $B$ is the insertion loss of a hydrophone or a delay coil. It is supposed that the loss due to fiber bends (bending loss) is predominant in the insertion loss of the fiber coil. All powers must be identical, and then the relation of adjacent FBGs is given by

$$
r_{n}=\frac{r_{n-1}}{\left(1-r_{n-1}\right)^{2} \alpha^{2} \beta^{2}} .
$$

Figure 2(b) shows the reflectance distribution which is calculated using Eq. (2) at $R_{1}, A$ and $B$ of $-30,0.02$ and 0.9 $\mathrm{dB}$, respectively. Here, we adopt $R_{1}$ of $-30 \mathrm{~dB}$ tentatively and a length of $100 \mathrm{~m}$ between FBGs to ensure that the sensitivities of hydrophones in Fig. 2(a) are similar to the 


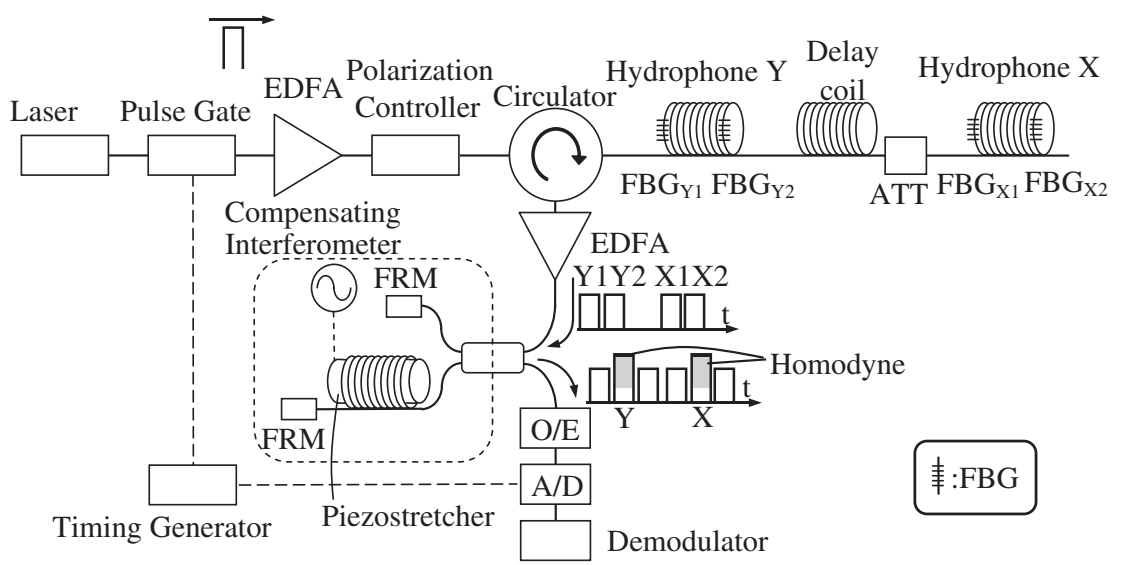

Fig. 1 Measurement setup of FBG-type hydrophone.

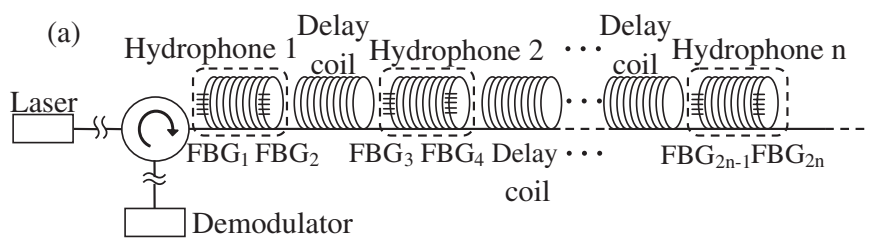

(b)

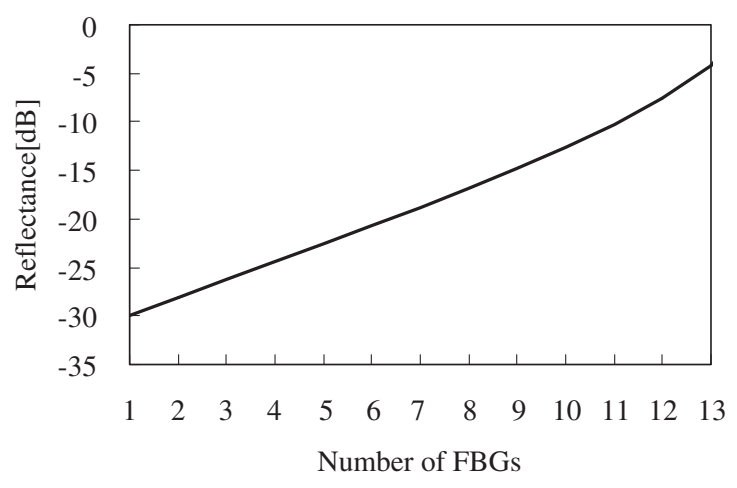

Fig. 2 Reflectance distribution of series of hydrophones: (a) schematic configuration and (b) calculation $\left(R_{1}=-30 \mathrm{~dB}, A=0.02 \mathrm{~dB}, B=0.9 \mathrm{~dB}\right)$.

prototype hydrophone [6]. Practical fibers are produced with losses below $0.2 \mathrm{~dB} / \mathrm{km}$ near $1,550 \mathrm{~nm} \mathrm{[10];} \mathrm{therefore,} \mathrm{we}$ assume $A=0.02 \mathrm{~dB}$. The measured insertion loss of hydrophone $\mathrm{Y}$ is $0.9 \mathrm{~dB}$; therefore, we consider this value as a representative loss of the fiber coil $(B=0.9 \mathrm{~dB})$. The schematic reflectance of FBGs gradually increases. Under the conditions above, with a series of 13 FBGs, namely, 6 hydrophones with TDM can be achieved theoretically, because the reflectance of the 14 th FBG is $1.9 \mathrm{~dB}(>0 \mathrm{~dB})$.

\section{Experiment}

Both hydrophones immersed in the sea are tested for sensitivity with TDM and a single configuration for comparison to demonstrate TDM. The design sensitivities for both hydrophones are $-140 \mathrm{~dB} \mathrm{re} 1 \mathrm{rad} / \mu \mathrm{Pa}$. The instrumentation setup for the measurement of FBG-type interferometric
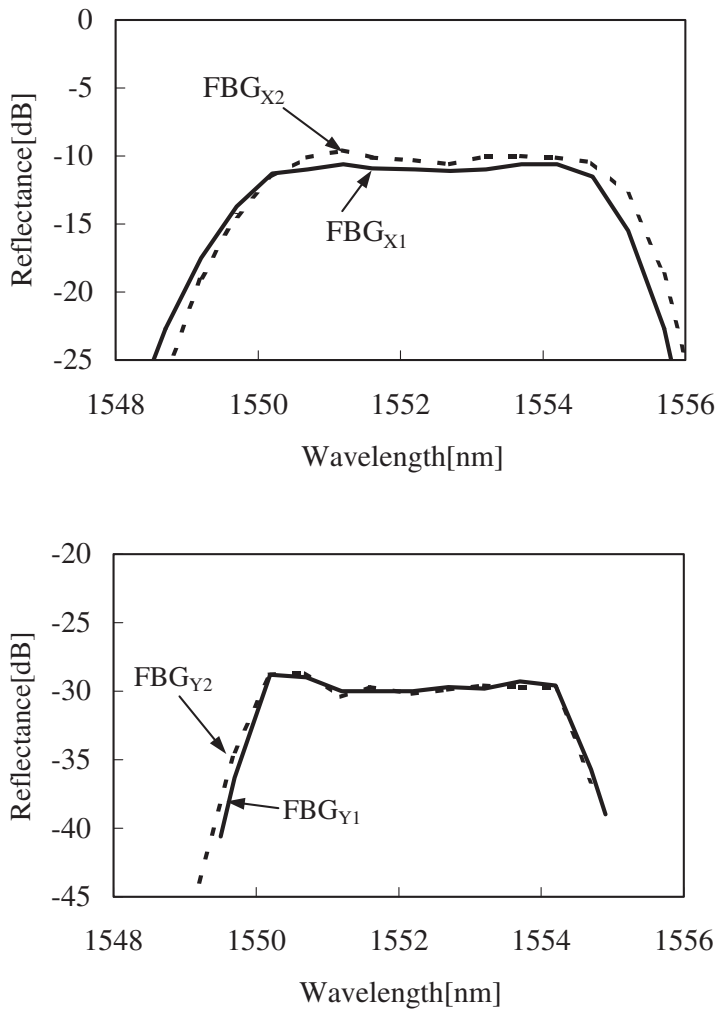

Fig. 3 Reflectance spectra of FBGs used in hydrophones.

hydrophones with TDM is shown in Fig. 1. When the sensitivity with a single configuration is tested, a hydrophone, an attenuator (ATT) and a delay coil are eliminated. Figure 3 shows the reflectance spectra of the FBGs used in two hydrophones. The Bragg wavelength ranges from 1,550 to $1,555 \mathrm{~nm}$ for each FBG. From the calculation mentioned above, we demonstrate the most basic TDM architecture, which consists of two hydrophones with their reflectances of approximately -30 and $-10 \mathrm{~dB}$. The reflectance of $-30 \mathrm{~dB}$ indicates the first hydrophone of an array. The reflectance of $-10 \mathrm{~dB}$ indicates the hydrophone near the end of the array. An ATT is inserted between hydrophones with an optical power loss to demonstrate a large TDM system. The 


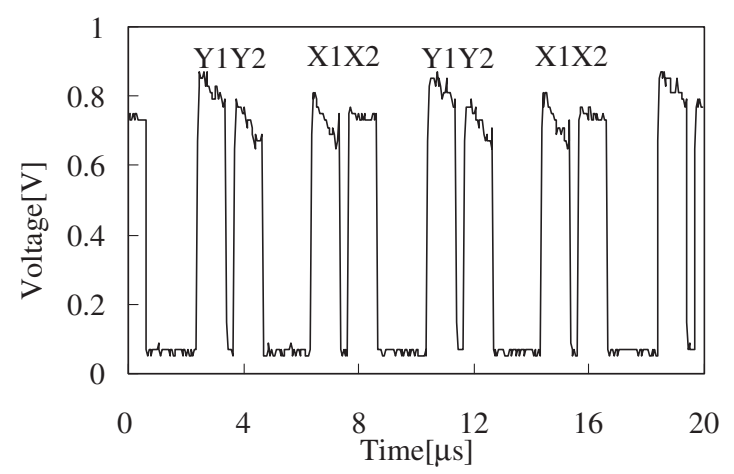

Fig. 4 Optical pulse train returning from hydrophones.

(a)

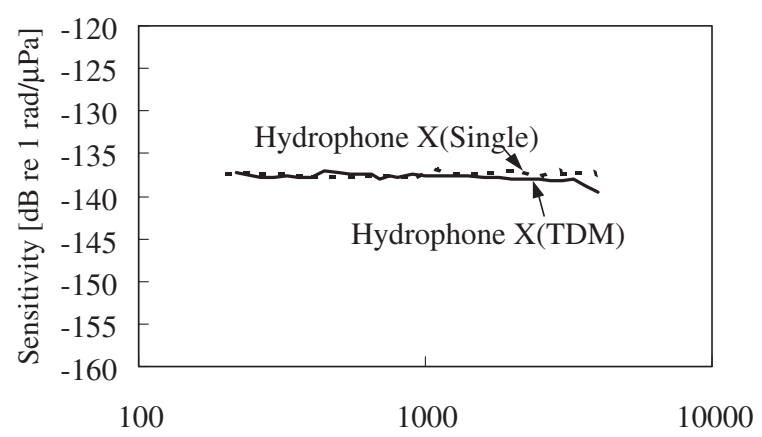

(b)

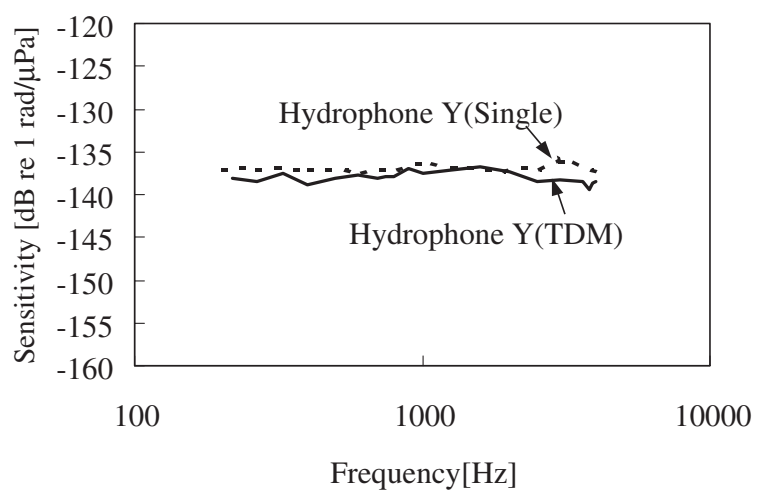

Fig. 5 Hydrophone sensitivity versus frequency with TDM and single configuration: (a) hydrophone $\mathrm{X}$ and (b) hydrophone Y.

calculated loss between these hydrophones is about $9.0 \mathrm{~dB}$, which is one-half of the difference between $\mathrm{FBG}_{2}(-28.2 \mathrm{~dB})$ and $\mathrm{FBG}_{11}(-10.3 \mathrm{~dB})$. Therefore, we set the insertion loss of a delay coil plus an ATT at $9.0 \mathrm{~dB}$, which corresponds to the calculated loss. A repetitive pulse signal is generated from a laser at a wavelength of $1,551.72 \mathrm{~nm}$.

The experimental results show the following. Figure 4 shows the $\mathrm{O} / \mathrm{E}$ converter output for the evaluation of the power of an optical pulse train without a compensating interferometer. This shows that similar powers are returned from the hydrophones, confirming the reliability of the proposed reflectance distribution design method.
The sensitivities of both hydrophones as a function of frequency are shown in Figs. 5(a) and 5(b). The mean sensitivities of hydrophone $\mathrm{X}$ with TDM and a single configuration are -137.8 and $-137.5 \mathrm{~dB}$, respectively, in the frequency range of $200 \mathrm{~Hz}$ to $4 \mathrm{kHz}$. Those of hydrophone $\mathrm{Y}$ are -138.0 and $-137.0 \mathrm{~dB}$, respectively. The experiment shows that the hydrophones exceed the performance target and that the difference in mean sensitivity between TDM and a single configuration is within $1.0 \mathrm{~dB}$. These results demonstrate the usefulness of the TDM configuration of the FBGtype hydrophone.

\section{Summary}

In this work, we propose the method of designing the reflectance distribution of FBGs to maximize the number of multiplexed hydrophones. The TDM configuration of FBGtype hydrophones is constructed, and an experiment is carried out. The experimental results show that 1) similar power pulses return from hydrophones based on the proposed reflectance distribution design method and 2) the sensitivities of hydrophones with TDM and a single configuration are similar. As a result, the effectiveness of the proposed method is exhibited.

To realize an FBG-type hydrophone array system, it is desirable that bending loss should be decreased and that we have to overcome PSF (which prevents us from realizing the implementation of an FBG-type hydrophone array system). Some methods of overcoming PSF are suggested [11,12], and we will discuss them in the near future.

\section{Acknowledgements}

The authors would like to thank Mr. R. Sato, Mr. Y. Nakajima and Mr. M. Minami of Oki Electric Industry Co., Ltd., for helpful comments and discussions.

\section{References}

[1] A. D. Kersey and A. Dandridge, "Distributed and multiplexed fiber-optic sensor systems,” J. IERE (Suppl.), 58, S99-S111 (1988).

[2] A. D. Kersey, M. A. Davis, H. J. Patrick, M. LeBlanc, K. P. Koo, C. G. Askins, M. A. Putnam and E. J. Friebele, "Fiber grating sensors," J. Lightwave Technol., 15, 1442-1463 (1997).

[3] G. A. Cranch, P. J. Nash and C. K. Kirkendall, "Large-scale remotely interrogated arrays of fiber-optic interferometric sensors for underwater acoustic applications," IEEE Sensors J., 3, 19-30 (2003).

[4] G. A. Cranch, R. Crickmore, C. K. Kinkendall, A. Bautista, K. Daley, S. Motley, J. Salzano, J. Latchem and P. J. Nash, "Acoustic performance of a large-aperture, seabed, fiber-optic hydrophone array," J. Acoust. Soc. Am., 115, 2848-2858 (2004).

[5] W. W. Morey, "Distributed fiber grating sensors," Proc. OFS '90, Sydney, Australia, pp. 285-288 (1990).

[6] C. Okawara, H. Shimamura, T. Uno and H. Uchida, "Acoustical property of the fiber optic hydrophone using fiber Bragg grating," Proc. Meet. Marine. Acoust. Soc. Jpn., pp. 117-118 (2004).

[7] G. A. Cranch and P. J. Nash, "Large-scale multiplexing of interferometric fiber-optic sensors using TDM and DWDM," $J$. Lightwave Technol., 19, 687-699 (2001).

[8] A. Dandridge, A. B. Tveten and T. G. Giallorenzi, "Homodyne 
demodulation scheme for fiber optic sensors using phase generated carrier," IEEE J. Quantum Electron., QE-18, 16471653 (1982).

[9] H. Arai, M. Fukazawa, R. Sato, K. Dobashi, Y. Kohama and M. Egawa, "Self-noise analysis of prototype fiber optic hydrophone using passive homodyne scheme," Proc. Spring. Meet. Acoust. Soc. Jpn., pp. 1167-1168 (1994).

[10] K. T. V. Grattan and B. T. Meggitt, Optical Fiber Sensor
Technology Fundamentals (Kluwer Academic Publishers, Boston, 2000), pp. 64-65.

[11] N. J. Frigo, A. Dandridge and A. B. Tveten, "Technique for elimination of polarization fading in fibre interferometers," Electron. Lett., 20, 319-320 (1984).

[12] J. P. Dakin, C. A. Wade and M. Henning, "Novel optical fibre hydrophone array using a single laser source and detector," Electron. Lett., 20, 53-54 (1984). 